\title{
Pricing American Options Using Transition Probabilities: A Dynamical Systems Approach
}

\author{
Rocio Elizondo ${ }^{1}$, Pablo Padilla ${ }^{2}$, Mogens Bladt ${ }^{3}$ \\ ${ }^{1}$ Directorate of Economic Studies, DGIE, Banco de México, Mexico City, Mexico \\ ${ }^{2}$ Department of Mathematics and Mechanics, IIMAS, UNAM, Mexico City, Mexico \\ ${ }^{3}$ Department of Probability and Statistics, IIMAS, UNAM, Mexico City, Mexico \\ Email: melizondo@banxico.org.mx, pablo@mym.iimas.unam.mx, bladt@sigma.iimas.unam.mx
}

Received 30 July 2015; accepted 15 October 2015; published 20 October 2015

Copyright (C) 2015 by authors and Scientific Research Publishing Inc.

This work is licensed under the Creative Commons Attribution International License (CC BY). http://creativecommons.org/licenses/by/4.0/

(c) (i) Open Access

\begin{abstract}
We give a new way to price American options by using Samuelson's formula. We first obtain the option price corresponding to a European option at time $t$, weighing it by the probability that the underlying asset takes the value $S$ at time $t$. We then use Samuelson's formula with this factor which is given by the solution of the Fokker-Planck (Kolmogorov) equation for the transition probability density. The main advantage of this approach is that we can systematically introduce the effect of macroeconomic factors. If a macroeconomic framework is given by a dynamical system in the form of a set of ordinary differential equations we only have to solve a partial differential equation for the transition probability density. In this context, we verify, for the sake of consistency, that this formula coincides with the Black-Scholes model and compare several numerical implementations.
\end{abstract}

\section{Keywords}

American Options, Fokker-Planck, Black-Scholes, Samuelson, Probability Density Function

\section{Introduction}

There are several methods for pricing European and American options [1]-[17]. We are interested in different ways to price American options that are flexible enough, so that they can be useful in nonstandard situations, or offer alternatives in standard settings.

Our main goal is to present a new way to price American options, which allows us to introduce the effect of 
macroeconomic factors that affect the financial market so we can introduce different dynamics for the underlaying asset and generalize more complex processes such as Levy processes.

This new formula does not pretend to improve on the existent methods in the context of the Black-Scholes model. We want to show that it is consistent with it. In fact it is equivalent to a previous one given by Carr, Jarrow and Minery [5]. The most interesting feature of this method is that it explicitly introduces the transition probability as a solution to a Fokker-Planck (Kolmogorov) equation. This allows us to consider more general situations when the underlaying asset does not follow a log-normal process.

We propose an extension of Samuelson's formula for American type contingent claims, because one of the most interesting aspects of this formula is that it does not make explicit reference to the risk neutral distribution, but the physical or observed one (see Section 2 below).

For the fixed underlaying asset value $S$ we propose that the American option price is:

$$
V_{A}(S, t)=E_{\rho}\left[V_{E u}(S, t)\right]=\int_{t}^{T_{S}} \rho_{n}(S, \tau) V_{E u}(S, \tau) \mathrm{d} \tau, S>S_{f},
$$

where for each time $\tau$ of $S, \rho_{n}(S, \tau)$ is the normalized solution of the corresponding Fokker-Planck equation (for the probability density function), $V_{E u}(S, \tau)$ is the solution of the Black-Scholes equation for the European case, $S_{f}$ is the free boundary and $T_{S}$ corresponds to the expiration time at the free boundary, which depends on the location of the free boundary (for details see section 3).

For practical purposes, we present a theoretical example introducing the effect of macroeconomic factors in a derivative model, and for consistency, we prove that this formula satisfies the Black-Scholes inequality for American options in the log-normal case.

In what follows we define $S_{t}$ : The underlaying asset price at time $t . E$ : Exercise price or maturity price. $T$ : Exercise time or maturity time. $T_{S}$ : Time to early exercise (on the free boundary). $t$ : Current time. $r$ : Free risk interest rate. $\sigma$ : Underlaying asset volatility. $V_{E u}(S, t)$ : European option price. $C_{E u}(S, t)$ : European call option price. $P_{E u}(S, t)$ : European put option price. $P_{A}(S, t)$ : American put option price. $C_{A}(S, t)$ : American call option price. $V_{A}(S, t)$ : American option price.

To value European type options there are explicit and standard formulas as Black-Scholes formula [14], Samuelson formula [18], an actuarial approach [2], Merton's theory of rational option pricing [9], etc. However, to value an American options there are not closed formulas. Nowadays, there are several representations to approximate the value of American options. In general, there are not closed expressions except in special situations. In the case that a formula exists, it can be very complex. The best way to price American options in practice is to use numerical methods (explicit, implicit, finite differences, between others) although they can be complicated, too.

The options are typically used for development of structural products, speculation strategies and hedging. Most of the options traded in the international markets are of American type.

For consistency, our objective is to prove analytically that (1) also coincides with standard approach in the literature as the Black-Scholes formula. We also compare its numerical implementation with the existent numerical methods such as: binomial trees, explicit and implicit partial differential equations methods.

In (1), we assume that we know a priori the location (or some approximation) of the free boundary. Existing methodologies both analytical and numerical can be used to approximate well the free boundary.

This article is structured in the following way. In the second section, we describe the Kolmogorov equations, which represents the transition probability density function in (1). In the third section, we present the formula to price the American options, as well as, some numerical examples and compare with others numerical methods. Finally, we give some conclusions and open problems.

\section{Preliminaries}

To understand the mechanism of our approach, we are going to describe the Kolmogorov backwards equation and Kolmogorov forward equation (Fokker-Planck equation). They are important tools for finding the probability density function.

\section{The Transition Probability Density Function}

We derive an equation for the probability density function of a random variable defined by a stochastic differential equation (SDE). This density describes the temporal evolution of the state, $x$, of the phenomenon 
under study.

We introduce a general random variable $x$, which satisfies the SDE:

$$
\mathrm{d} x=f(x, t) \mathrm{d} t+g(x, t) \mathrm{d} W,
$$

where $f$ and $g$ are any continuously differentiable functions of $x$ and $t$, and $W$ is the Wiener process or Brownian motion.

Then, the transition probability density function is denoted by $p\left(x, t \mid x^{*}, t^{*}\right)$ with $t \leq t^{*}, x^{*}$ and $t^{*}$ the future values of $x$ and $t$ respectively.

It is well know that $p$ satisfies:

$$
\frac{\partial p}{\partial t^{*}}=\frac{1}{2} \frac{\partial^{2}\left(g^{2}\left(x^{*}, t^{*}\right) p\right)}{\partial\left(x^{*}\right)^{2}}-\frac{\partial\left(f\left(x^{*}, t^{*}\right) p\right)}{\partial t^{*}},
$$

with the initial condition

$$
p\left(x, t ; x^{*}, t^{*}\right)=\delta\left(x^{*}-x\right) \text { and } t=t^{*},
$$

where $\delta(\cdot)$ is the Dirac delta function.

Conversely, if we know the value $x^{*}$ at time $t^{*}$, then we can determine an equation for the probability density function for $x$ at an earlier time $t$. This is called the backwards Kolmogorov equation and it is given by

$$
\frac{\partial p}{\partial t}=-\frac{1}{2} g^{2}(x, t) \frac{\partial^{2} p}{\partial x^{2}}-f(x, t) \frac{\partial p}{\partial x},
$$

with final condition given by

$$
p\left(x, t ; x^{*}, t^{*}\right)=\delta\left(x-x^{*}\right) \text { and } t=t^{*} .
$$

For a detailed presentation of these subjects see [14] [19]-[22].

\section{Alternative Formula to Estimate American Options}

We are going to evaluate an American option applying Samuelson's formula ([2] [18]), using the transition probability density to generate the function corresponding to the physical measure as the solution of a FokkerPlanck type equation ([19] [20] [23]-[25]). For consistency, we are going to prove this new formula recovers the traditional Black-Scholes results in the log-normal case ([10] [12] [14] [23] [26]-[28]) for the price of an American put option. We are also going to compare this proposal with specific examples estimated with the tree methods (Cox, Ingersoll and Rubinstein) and the finite differences, explicit and implicit methods.

The main idea behind this formulation is that we can use the Fokker-Planck equation in more general or complex problems in order to incorporate the effect of macroeconomic factors to several models of financial derivatives and credit risk, where we do not know a priori the real distribution of the density and where the underlying asset does not follow a log-normal distribution.

\subsection{A General Formula for Models Incorporating Macroeconomic Variables}

The importance of considering macroeconomic factors in pricing standard or credit risk derivatives is that they considerably affect their value (see [29]).

Our idea is to extend this model to the case where the parameters may depend directly on different macroeconomic factors or indices. In order to do this we generate the probability density function through the Fokker-Planck equation, which will allow us to systematically introduce the effect of macroeconomic factors on the option-pricing model.

We have a financial model that takes into account the effect of macroeconomic factors. Suppose we want to calculate the financial derivative value with underlying asset $S$, but the parameters $\mu$ and $\sigma$ are dependent on $n$ macroeconomic factors.

We consider that a macroeconomic environment is formed by $n$ variables,

$X=\left(X_{1}, X_{2}, \cdots, X_{n}\right)$, which are represented by the dynamics: 


$$
\mathrm{d} X=G(X, t) \mathrm{d} t+\alpha F(X) \mathrm{d} \tilde{W},
$$

where $G(\cdot)$ is a function that depends on macroeconomic factors and time, $\alpha$ is a constant and $\mathrm{d} \tilde{W}$ represents a Brownian motion ${ }^{1}$.

Now, we suppose that the underlying asset, in general, is governed by the stochastic differential equation:

$$
\mathrm{d} S_{t}=\mu\left(X_{1}, \cdots, X_{n}, t\right) S_{t} \mathrm{~d} t+\sigma\left(X_{1}, \cdots, X_{n}, t\right) S_{t} \mathrm{~d} W .
$$

In this case, the way to introduce the macroeconomic factors and time is through the return rate $\mu$, the volatility $\sigma$ and the free risk interest risk $r$. In this step it is necessary to calibrate the model in such a way as to determine the effect that such factors have on the parameters.

On the other hand, we calculate the transition probability through the Fokker-Planck equation, but generalized to $n$ variables ${ }^{2}$. In this case, $\rho(X, t)$ is the probability of finding the system in state $X$ at time $t$.

The advantage of using this equation lies in being able to represent concisely the dynamics of the system of equations by a single partial differential equation, via the transition probability function.

Finally, we can apply Samuelson formula or our formula to obtain the price of American or European options:

$$
V_{A}(S, t)=\mathrm{e}^{-r t} E_{\rho}\left[g\left(\frac{S_{t}}{E\left(S_{t}\right)} S_{0} \mathrm{e}^{r t}\right)\right]=\mathrm{e}^{-r t} \int_{t}^{T_{S}} g\left(\frac{S}{E\left(S_{\tau}\right)} S_{0} \mathrm{e}^{r \tau}\right) \rho(S, \tau) \mathrm{d} \tau .
$$

With $S \in[0, \infty]$ the underlying asset price, $0 \leq t \leq T_{S} \leq T$ is the early exercise time on the free boundary.

The Fokker-Planck equation can be used in credit risk and present net value problems (see [30]), among many other cases where the transition probability follows more general processes, such as Lévy process.

We will consider the case of an American option on a single underlying asset following a log-normal process. Here $\mu$ and $\sigma$ are constant.

\subsection{Alternative Formula for Pricing American Options}

Introducing the alternative formula for estimating American options, we define the American option price without dividends in the following way. For a fixed $S$, we have:

$$
\begin{aligned}
V_{A}(S, t) & =E_{\rho}\left[V_{E u}(S, t)\right]=\text { Put }= \begin{cases}\int_{t}^{T_{S}} \rho_{n}(S, \tau) V_{E u}(S, \tau) \mathrm{d} \tau, & S>S_{f} ; \\
P O, & S \leq S_{f},\end{cases} \\
& =\text { Call }= \begin{cases}\int_{t}^{T_{S}} \rho_{n}(S, \tau) V_{E u}(S, \tau) \mathrm{d} \tau, & S<S_{f} ; \\
P O, & S \geq S_{f},\end{cases}
\end{aligned}
$$

with

$$
P O(S, t)= \begin{cases}S-E, & \text { call option; } \\ E-S, & \text { put option, }\end{cases}
$$

where $S \in[0, \infty], S_{f}$ is the free boundary, $0<t \leq T_{S}$ is current time, $0 \leq T_{S} \leq T$ is the early exercise time on the free boundary ${ }^{3}, T$ is the maturity time (to see the relation between the times $T$ and $T_{S}$ see Figure 1), $\rho_{n}(S, t)$ is the normalized probability that the asset takes the value $S$ at time $t$ and $V_{E u}$ is the analytical solution to the Black-Scholes equation, namely:

${ }^{1}$ If the system depends on one macroeconomic factor such that $X=r$, the risk free interest rate, then we can consider the dynamics for $r$ given by the CIR or a Vasicek model, namely, $\mathrm{d} r=a(b-r) \mathrm{d} r+\alpha r^{\beta} \mathrm{d} \tilde{W}$.

${ }^{2}$ If $F\left(X_{i}\right)=1$ for all $i$, then the Fokker-Planck equation is given by

$$
\frac{\partial \rho}{\partial t}=\operatorname{div}(G \rho)+\sqrt{2 \alpha} \Delta \rho
$$

with $\operatorname{div}(G \rho)=\sigma \sum \frac{\partial(G \rho)}{\partial X_{i}}$ and $\Delta \rho=\sum \frac{\partial^{2} \rho}{\partial X_{i}^{2}}$.

${ }^{3}$ Note that when we say that $T_{S}$ is the time to expiration, we mean the time corresponding to the free boundary, i.e., for each $S$ there is a time $T_{S}$ on the free boundary. 


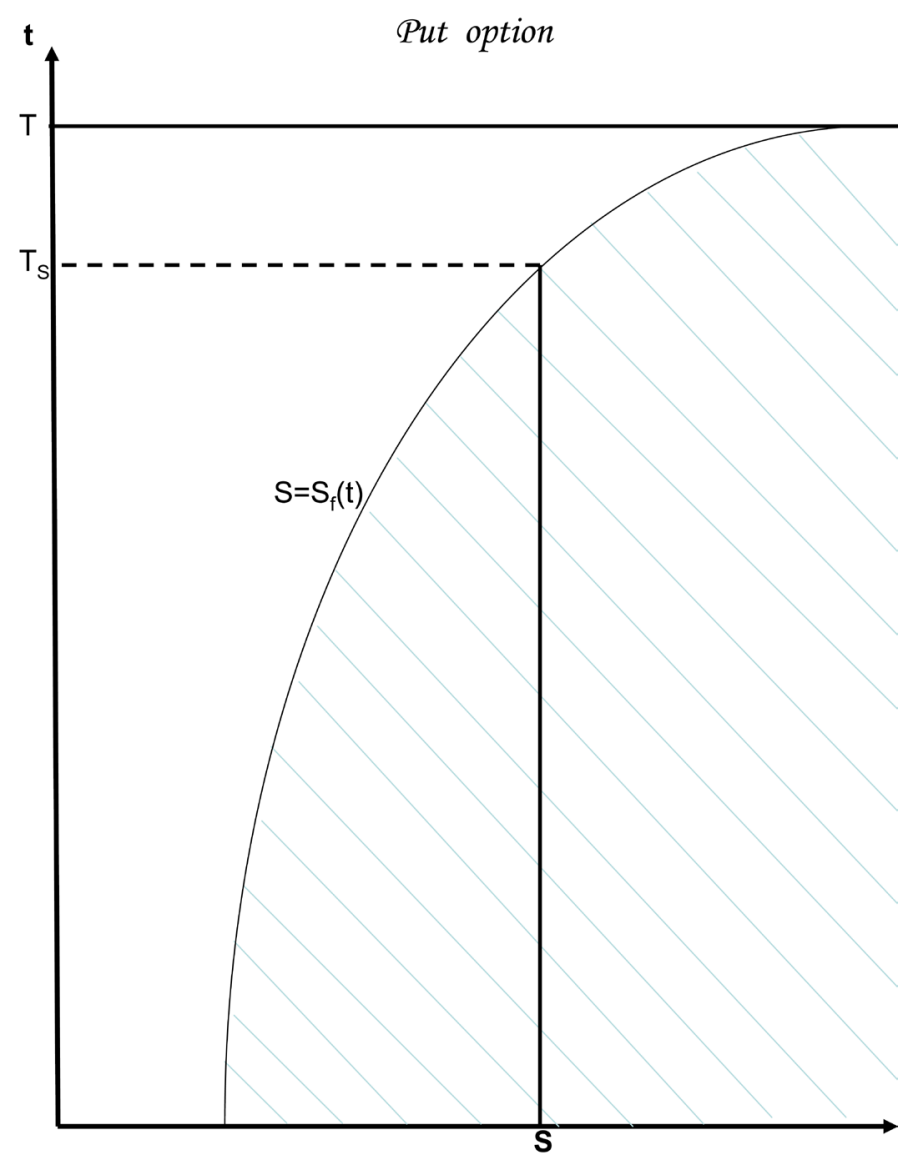

Figure 1. Relationship between the times $T$ and $T_{S}$.

$$
V_{E u}(S, t)= \begin{cases}S N\left(d_{1}\right)-E \mathrm{e}^{-r t} N\left(d_{2}\right), & \text { call option; } \\ E \mathrm{e}^{-r t} N\left(-d_{2}\right)-S N\left(-d_{1}\right), & \text { put option, }\end{cases}
$$

where

$$
\begin{gathered}
d_{1}=\frac{\ln \left(\frac{S}{E}\right)+\left(r+\sigma^{2} / 2\right) t}{\sigma \sqrt{t}} \\
d_{2}=\frac{\ln \left(\frac{S}{E}\right)+\left(r-\sigma^{2} / 2\right) t}{\sigma \sqrt{t}}=d_{1}-\sigma \sqrt{t},
\end{gathered}
$$

and $N(\cdot)$ is the cumulative normal distribution function with zero mean and standard deviation equal to one.

The consistency conditions are:

$$
V_{A}(0, t)= \begin{cases}0, & \text { call option; } \\ E, & \text { put option, }\end{cases}
$$

and

$$
V_{A}(S, t) \rightarrow_{S \rightarrow \infty} \begin{cases}S, & \text { call option; } \\ 0, & \text { put option. }\end{cases}
$$

There is another alternative formula to estimate American options where the integration is with respect to 
time too (see [5]).

Before proceeding with the details of our alternative formula it is necessary to define the location of the free boundary. We present the results we need in the following section.

\section{Free Boundary}

The problem of finding the American option value translates into finding a solution to a free boundary problem. The strategy for approaching the solution is to locate the free boundary, $S_{f}$.

So if $V_{A}$ is continuous and monotone, there exists a value $0<S_{f}<E$, where the free boundary is located and $V_{A}=E-S$ for a put option and $V_{A}(S, t)=S-E$ for a call option.

For a Call option $\left(C_{A}\right)$ :

$$
\left\{\begin{array}{lc}
S<S_{f}, & \text { no early exercise; } \\
S \geq S_{f}, & \text { exercise. }
\end{array}\right.
$$

Put option $\left(P_{A}\right)$ :

$$
\left\{\begin{array}{lc}
S>S_{f}, & \text { no early exercise; } \\
S \leq S_{f}, & \text { exercise. }
\end{array}\right.
$$

The curve $S_{f}$ divides the plane $t S$ in two parts as shown in Figure 2. The shaded parts of both graphs, represent the domain where the option, call $\left(S<S_{f}\right)$ or put $\left(S>S_{f}\right)$, is not exercised and its value is given by:

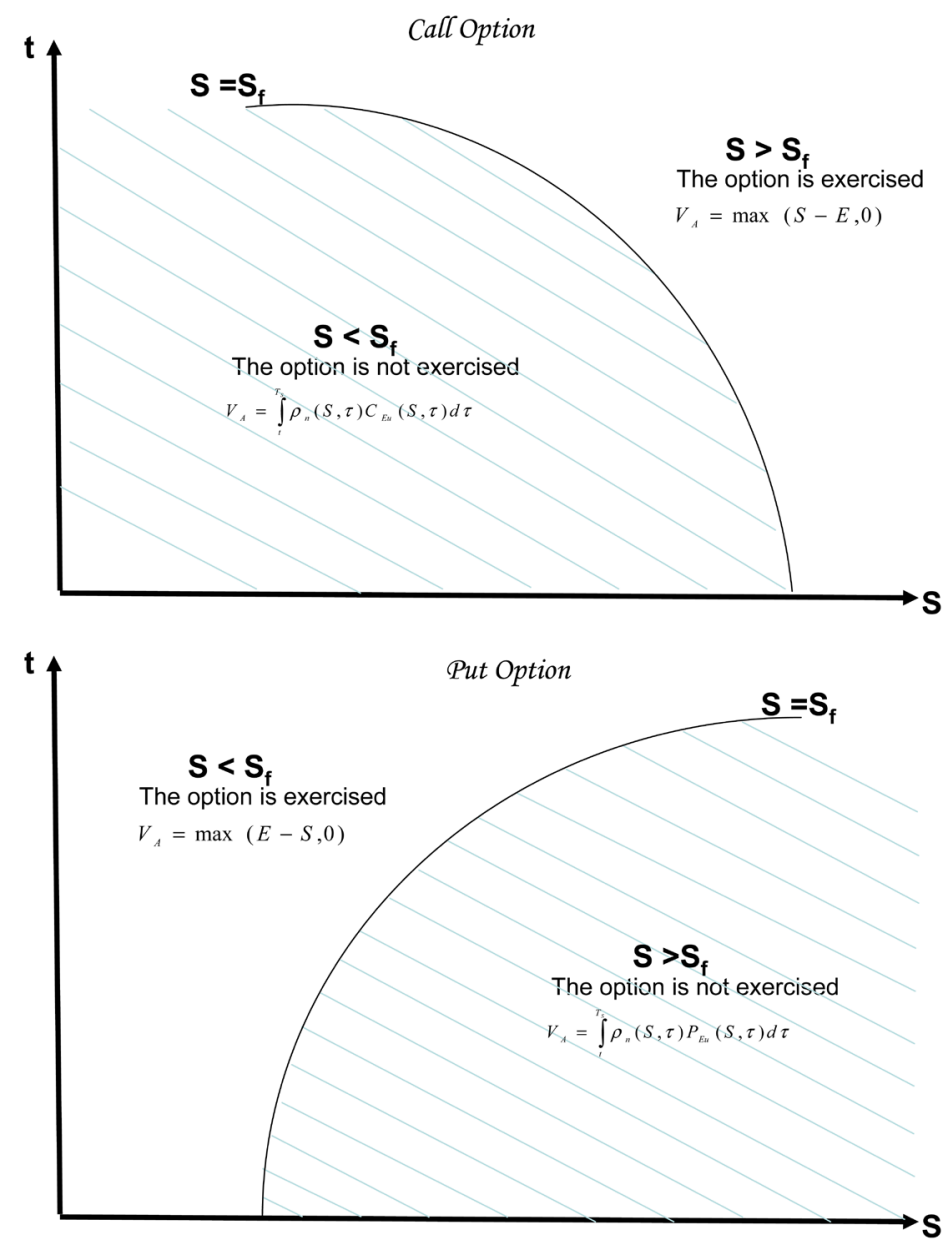

Figure 2. Region of solution of an American option. 


$$
V_{A}= \begin{cases}\int_{t}^{T_{S}} \rho_{n} C_{E u}(S, \tau) \mathrm{d} \tau, & \text { call option; } \\ \int_{t}^{T_{S}} \rho_{n} P_{E u}(S, \tau) \mathrm{d} \tau, & \text { put option. }\end{cases}
$$

The region outside the curve, $S=S_{f}$, is where the option is exercised. This region for the call option is $S>S_{f}$ and for the put option is $S<S_{f}$, and its value is given by their respective payoff function, $P O$.

For a put option, a point outside the curve in the upper region is given by $\left(S^{*}, t\right)$ for $0<t^{*}<T$ and $S<S_{f}$. A point on the curve is represented by $\left(S_{f}, t\right)$ for $0<t<T_{S}$ and $S=S_{f}$. Finally, a point outside of the curve in the lower region is given by $(S, t)$ for $0<t<T_{S}$ and $S>S_{f}$. The case of a call option is similar, we only have to invert de inequalities corresponding to $S$ with respect to $S_{f}$.

This determines the region of solution for an American option.

We now define the price of an American option in terms of the price of a European option at each time $t$, where $t \in\left[0, T_{S}\right]$, multiplied by a normalizing weight $\rho$, representing the probability of attaining $S$ at time $t$. Finally these values are integrated with respect to time.

Mathematically, for each $t$, the weight $\rho$, corresponds to the transition probability density function, which is determined by the Fokker-Planck equation as follows:

$$
\frac{\partial \rho}{\partial t}=\frac{1}{2} \frac{\partial^{2}\left(\sigma^{2} S^{2} \rho\right)}{\partial S^{2}}-\frac{\partial(r S \rho)}{\partial S},
$$

calculating the the partial derivatives we have:

$$
\frac{\partial \rho}{\partial t}=\frac{1}{2} \sigma^{2} S^{2} \frac{\partial^{2} \rho}{\partial S^{2}}+\left(2 \sigma^{2}-r\right) S \frac{\partial \rho}{\partial S}+\left(\sigma^{2}-r\right) \rho
$$

with boundary conditions:

$\rho(0,0)=0$. This condition is not necessary for the case of an American put option. ${ }^{4}$

$\rho(S, 0)=\delta\left(S-S_{0}\right)$ for $S \in[0, \infty)$, where $\delta$ is the Dirac delta.

$\rho\left(S_{f}, t\right)=\tilde{\rho}\left(S_{f}, t\right)$, where $S_{f}$ is the free boundary.

The Fokker-Planck equation with respect to $\tilde{\rho}(S, t)$ is defined in the rectangle $[0, S] \times[0, T]$ and has boundary conditions $\tilde{\rho}(0, t)=0$ and $\tilde{\rho}(S, t) \rightarrow 0$ when $S \rightarrow \infty$. In our alternative formula, the solution region is restricted by the curve resulting from the free boundary $S_{f}$.

$\rho(S, t) \rightarrow 0$ when $S \rightarrow \infty$.

Another condition of consistency but with respect to the parameter is $\rho(S, t) \rightarrow 0$ when $S_{0} \rightarrow \infty$.

Remark 1 It is very important to keep in mind that the density $\rho$ does not satisfy a free boundary problem. The free boundary is given for the option price, namely, the domain in which we define $\rho$ is well determined and does not depend on $\rho$ itself.

Remark 2 The Fokker-Planck equation with respect to $\rho$ has an explicit solution in the log-normal case (Black-Scholes) $)^{5}$, and that solution is given as follows.

Let $S_{0}$ be the initial value of the underlying asset and $t_{0}$ the initial time. $S$ and $t$ are the future values. The probability that the underlying asset takes the value of $S$ at time $t$, given the initial time $t_{0}$ and the corresponding value $S_{0}$ is:

$$
\tilde{\rho}\left(S_{0}, t_{0} ; S, t\right)=\frac{1}{\sigma S \sqrt{2 \pi\left(t-t_{0}\right)}} \mathrm{e}^{-\left[\log \left(\frac{S}{S_{0}}\right)-\left(\mu-\frac{1}{2} \sigma^{2}\right)\left(t-t_{0}\right)\right]^{2} \frac{1}{2 \sigma^{2}\left(t-t_{0}\right)}} .
$$

This function satisfies the Fokker-Planck partial differential equation and the backwards Kolmogorov equation.

Remark 3 The normalization for the probability density function is given by:

$$
\int_{t}^{T_{S}} \rho_{n}(S, \tau) \mathrm{d} \tau=1
$$

\footnotetext{
${ }^{4}$ Since the origin is contained in the interior of the early exercise region.

${ }^{5}$ In general, the Fokker-Planck equation does not have explicit solutions.
} 
with

$$
\rho_{n}(S, t)=\frac{\rho(S, t)}{\int_{t}^{T_{S}} \rho(S, \tau) \mathrm{d} \tau} .
$$

Remark 4 Exercising an American call option before expiry is not optimal, because $C_{A}(S, t)>\max (S-E, 0)$ (the benefit of exercising the option is less than keeping the option), so the optimal time to exercise these options is at its maturity, therefore the value of a European option is equal to its American counterpart. This is a well known fact (see in [14] [31] [32]).

According to this last remark, the interesting case to study is that of an American put option, hence from now on we will focus on this case. This is not valid anymore if we consider that the $S$ can give dividends.

\subsection{American Put Option Formula}

Let $P_{E u}$ and $P_{A}$ be the European and American put option prices respectively, then we claim that the price of an American put option is

$$
P_{A}(S, t)= \begin{cases}\int_{t}^{T_{S}} \rho_{n}(S, \tau) P_{E u}(S, \tau) \mathrm{d} \tau, & S>S_{f} ; \\ \max (E-S, 0), & S \leq S_{f} .\end{cases}
$$

The probability density function $\rho$ satisfies the conditions mentioned above.

The consistency conditions of an American put option are:

$$
P_{A}(0, t)=\max (E-0,0)=E .
$$

$P_{A}(S, t) \rightarrow 0$ when $S \rightarrow \infty$. Indeed

$$
\begin{aligned}
& \lim _{S \rightarrow \infty} P_{A}(S, t)=\lim _{S \rightarrow \infty} \int_{t}^{T_{S}} \rho_{n}(S, \tau) P_{E u}(S, \tau) \mathrm{d} \tau \leq \int_{t}^{T_{S}} \lim _{S \rightarrow \infty} \rho_{n}(S, t) \lim _{S \rightarrow \infty} P_{E u}(S, \tau) \mathrm{d} \tau \\
& \rightarrow \int_{t}^{T_{S}} \rho_{n}(\infty, 0)(0) \mathrm{d} \tau=0 .
\end{aligned}
$$

This follows from Fatou's lemma. Notice that $\int_{0}^{t} \rho_{n}(S, \tau) \mathrm{d} \tau$ is bounded when $S \rightarrow \infty$, and clearly $P_{A} \geq 0$.

$$
\text { Moreover } \frac{\partial P_{A}\left(S_{f}, t\right)}{\partial S}=-1 \text { for } S=S_{f} \text {. }
$$

This can be proved using the fact that the price of a put option near the free boundary $S_{f}$ and considering that $S_{f}<E$, can be written as: ${ }^{6}$

with $d=\operatorname{dist}\left(S, S_{f}\right)$ (see [21]). Then

$$
P_{E u}=E-S+o\left(d\left(S, S_{f}\right)\right) \text {, }
$$

$$
\begin{aligned}
P_{A} & =\int_{t}^{T_{S}} \rho_{n}(S, \tau) P_{E u}(S, \tau) \mathrm{d} \tau=\int_{t}^{T_{S}} \rho_{n}(S, \tau)[E-S+o(d)] \mathrm{d} \tau \\
& =(E-S) \int_{t}^{T_{S}} \rho_{n}(S, \tau) \mathrm{d} \tau+o(d) \int_{t}^{T_{S}} \rho_{n}(S, \tau) \mathrm{d} \tau=(E-S)+o(d) .
\end{aligned}
$$

If we calculate the partial derivative with respect to $S$ of the Equation (20) we get:

$$
\frac{\partial P_{A}(S, t)}{\partial S}=\frac{\partial[E-S]}{\partial S}+o(1)
$$

and consequently we have:

$$
\frac{\partial P_{A}\left(S_{f}, t\right)}{\partial S}=\lim _{S \rightarrow S_{f}}\left[\frac{\partial(E-S)}{\partial S}+o(1)\right]=-1 .
$$

${ }^{6}$ If $V_{A}$ is continuous and monotone, there exist $S_{f}$ such that $0<S_{f}<E$. Otherwise, $S_{f}>E$, is exercised the option. For more details can consult [14]. 
The next subsection shows, for consistency, that the alternative formula satisfies the Black-Scholes inequality.

\subsection{Consistency of the Formula with the Black-Scholes Approach}

The advantage of this new formula, provided we know the location of the free boundary, is that we only need to asses the value of the function $\rho$, because the solution of the European option is known analytically by the Black-Scholes equation. Moreover we can introduce systematically the effect of macroeconomic factors within the valuation [30]. In general, the best way to obtain the function $\rho$ is numerically. First we verify, for the sake of consistency, that this new way of pricing American options is equivalent to the familiar Black-Scholes approach in the log-normal case.

Remember that the Black-Scholes inequality for American options (see [14]) is given by:

$$
\frac{\partial V_{A}}{\partial t}+\frac{1}{2} \sigma^{2} S^{2} \frac{\partial^{2} V_{A}}{\partial S^{2}}+r S \frac{\partial V_{A}}{\partial S}-r V_{A} \leq 0
$$

Let $\bar{\tau}=\tau+t$, then

$$
V_{A}=\int_{t}^{T_{S}} \rho_{n}(S, \bar{\tau}) V_{E u}(S, \bar{\tau}) \mathrm{d} \bar{\tau}=\int_{0}^{T_{S}-t} \rho_{n}(S, \tau+t) V_{E u}(S, \tau+t) \mathrm{d} \tau .
$$

Without loss of generality and to simplify the calculations, we write

$$
\rho_{n}(S, \tau+t)=\rho, \quad V_{E u}(S, \tau+t)=V_{E u} \text { and } 0 \leq t \leq T_{S} .
$$

Afterwards, we calculate the partial derivatives appearing in (16) and (23), and considering the value of $\partial \rho / \partial t,{ }^{7}$ inequality (22) is transformed in:

$$
\begin{aligned}
& \frac{\partial V_{A}}{\partial t}+\frac{1}{2} \sigma^{2} S^{2} \frac{\partial^{2} V_{A}}{\partial S^{2}}+r S \frac{\partial V_{A}}{\partial S}-r V_{A}=\int_{0}^{T_{S}-t} \rho^{2} V_{E u} \mathrm{~d} \tau-\underline{\rho\left(S, T_{S}-t\right) V_{E u}\left(S, T_{S}\right)} \\
& -r S \int_{0}^{T_{S}-t} \frac{\rho}{I_{\rho}}\left(\int_{t+\tau}^{T_{S}} \frac{\partial \rho}{\partial S} \mathrm{~d} k\right) V_{E u} \mathrm{~d} \tau-r S \int_{0}^{T_{S}-t} \frac{\rho^{2}}{\left(I_{\rho}\right)^{2}} \frac{\partial T_{S}}{\partial S} V_{E u} \mathrm{~d} \tau+r S \frac{\rho}{I_{\rho}} \frac{\partial T_{S}}{\partial S} V_{E u} \\
& -\sigma^{2} S^{2} \int_{0}^{T_{S}-t} \frac{1}{\left(I_{\rho}\right)^{2}} \frac{\partial \rho}{\partial S}\left(\int_{t+\tau}^{T_{S}} \frac{\partial \rho}{\partial S} \mathrm{~d} k\right) V_{E u} \mathrm{~d} \tau+\sigma^{2} S^{2} \int_{0}^{T_{S}-t} \frac{\rho}{\left(I_{\rho}\right)^{2}}\left(\int_{t+\tau}^{T_{S}} \frac{\partial \rho}{\partial S} \mathrm{~d} k\right)^{2} V_{E u} \mathrm{~d} \tau \\
& -\frac{1}{2} \sigma^{2} S^{2} \int_{0}^{T_{S}-t} \frac{\rho}{I_{\rho}}\left(\int_{t+\tau}^{T_{S}} \frac{\partial^{2} \rho}{\partial S^{2}} \mathrm{~d} k\right) V_{E u} \mathrm{~d} \tau-\sigma^{2} S^{2} \int_{0}^{T_{S}-t} \frac{\rho}{I_{\rho}} \frac{\partial \rho}{\partial S} \frac{\partial T_{S}}{\partial S} V_{E u} \mathrm{~d} \tau+2 \sigma^{2} S^{2} \int_{0}^{T_{S}-t} \frac{\rho^{2}}{I_{\rho}} \\
& \times \frac{\partial T_{S}}{\partial S}\left(\int_{t+\tau}^{T_{S}} \frac{\partial \rho}{\partial S} \mathrm{~d} k\right) V_{E u} \mathrm{~d} \tau-\sigma^{2} S^{2} \int_{0}^{T_{S}-t} \frac{\rho}{I_{\rho}} \frac{\partial T_{S}}{\partial S} V_{E u} \mathrm{~d} \tau+\sigma^{2} S^{2} \int_{0}^{T_{S}-t} \frac{\rho^{3}}{\left(I_{\rho}\right)^{3}}\left(\frac{\partial T_{S}}{\partial S}\right)^{2} V_{E u} \mathrm{~d} \tau \\
& \underline{-\frac{1}{2} \sigma^{2} S^{2} \int_{0}^{T_{S}-t} \frac{\rho^{2}}{\left(I_{\rho}\right)^{2}} \frac{\partial^{2} T_{S}}{\partial S^{2}} V_{E u} \mathrm{~d} \tau+\sigma^{2} S^{2} \int_{0}^{T_{S}-t} \frac{1}{I_{\rho}} \frac{\partial \rho}{\partial S} \frac{\partial V_{E u}}{\partial S} \mathrm{~d} \tau-\sigma^{2} S^{2} \int_{0}^{T_{S}-t} \frac{\rho}{\left(I_{\rho}\right)^{2}}\left(\int_{t+\tau}^{T_{S}} \frac{\partial \rho}{\partial S} \mathrm{~d} k\right)} \\
& \times \frac{\partial V_{E u}}{\partial S} \mathrm{~d} \tau-\sigma^{2} S^{2} \int_{0}^{T_{S}-t} \frac{\rho^{2}}{\left(I_{\rho}\right)^{2}} \frac{\partial T_{S}}{\partial S} \frac{\partial V_{E u}}{\partial S} \mathrm{~d} \tau+\sigma^{2} S^{2} \frac{1}{I_{\rho}} \frac{\partial \rho}{\partial S} \frac{\partial T_{S}}{\partial S} V_{E u}-\sigma^{2} S^{2} \frac{\rho}{\left(I_{\rho}\right)^{2}} \frac{\partial T_{S}}{\partial S} \\
& \times\left(\int_{t+\tau}^{T_{S}} \frac{\partial \rho}{\partial S} \mathrm{~d} k\right) V_{E u}-\sigma^{2} S^{2} \frac{\rho^{2}}{\left(I_{\rho}\right)^{2}}\left(\frac{\partial T_{S}}{\partial S}\right)^{2} V_{E u}+\sigma^{2} S^{2} \frac{\rho}{I_{\rho}} \frac{\partial V_{E u}}{\partial S} \frac{\partial T_{S}}{\partial S}+\frac{1}{2} \sigma^{2} S^{2} \frac{\rho}{I_{\rho}} \frac{\partial^{2} T_{S}}{\partial S^{2}} V_{E u} \leq 0,
\end{aligned}
$$

\footnotetext{
${ }^{7}$ We only have to observe that the Black-Scholes equation is backwards. Then, in order to make the consistent calculations, we have to take the backwards Kolmogorov equation for $\rho$. For this reason we consider the following equation (making the change of variable $\tau=T-t$ and abusing the notation, using again $t$ instead of $\tau$ ):
}

$$
\frac{\partial \rho}{\partial t}=-r S \frac{\partial \rho}{\partial S}-\frac{1}{2} \sigma^{2} S^{2} \frac{\partial^{2} \rho}{\partial S^{2}} .
$$


where $I_{\rho}=\int_{t}^{T_{S}} \rho(S, \bar{\tau}) \mathrm{d} \bar{\tau}=\int_{0}^{T_{S}-t} \rho(S, \tau+t) \mathrm{d} \tau$.

It is necessary to check that the left hand side of the inequality is less than or equal to zero, which we will do this next. It is straightforward to verify that all underlined terms are negative. We show that the positive terms, after an appropriate rescaling, are always of lower order than the negative ones. First we have to recall some important facts that will be useful in the proof. The term $\partial V_{E u} / \partial S$, is given by the Greek letter $\Delta$, which corresponds to the expression:

$$
\Delta P_{E u}=\frac{\partial P_{E u}}{\partial S}=N\left(d_{1}\right)-1 \text { with } d_{1}=\frac{\ln \left(S_{0} / E\right)+\left(r+\sigma^{2} / 2\right) T}{\sigma \sqrt{T}},
$$

which is negative for the European put option with a non-dividend-paying underlying asset and

$$
\Delta C_{E u}=\frac{\partial C_{E u}}{\partial S}=N\left(d_{1}\right) \text { with } d_{1}=\frac{\ln \left(S_{0} / E\right)+\left(r+\sigma^{2} / 2\right) T}{\sigma \sqrt{(}(T)} .
$$

This is always positive for a European call option with a non-dividend-paying asset (see [14] y [35]). We show in Figure 3 the variation of delta of a call and put option with respect to the underlaying asset, as well as the variation of the delta with respect to maturaty time.

Hence it follows that:

$$
S_{1} \leq S_{2} \Rightarrow P_{E u}\left(S_{1}, t\right) \geq P_{E u}\left(S_{2}, t\right) \Rightarrow \frac{\partial P_{E u}}{\partial S} \leq 0
$$
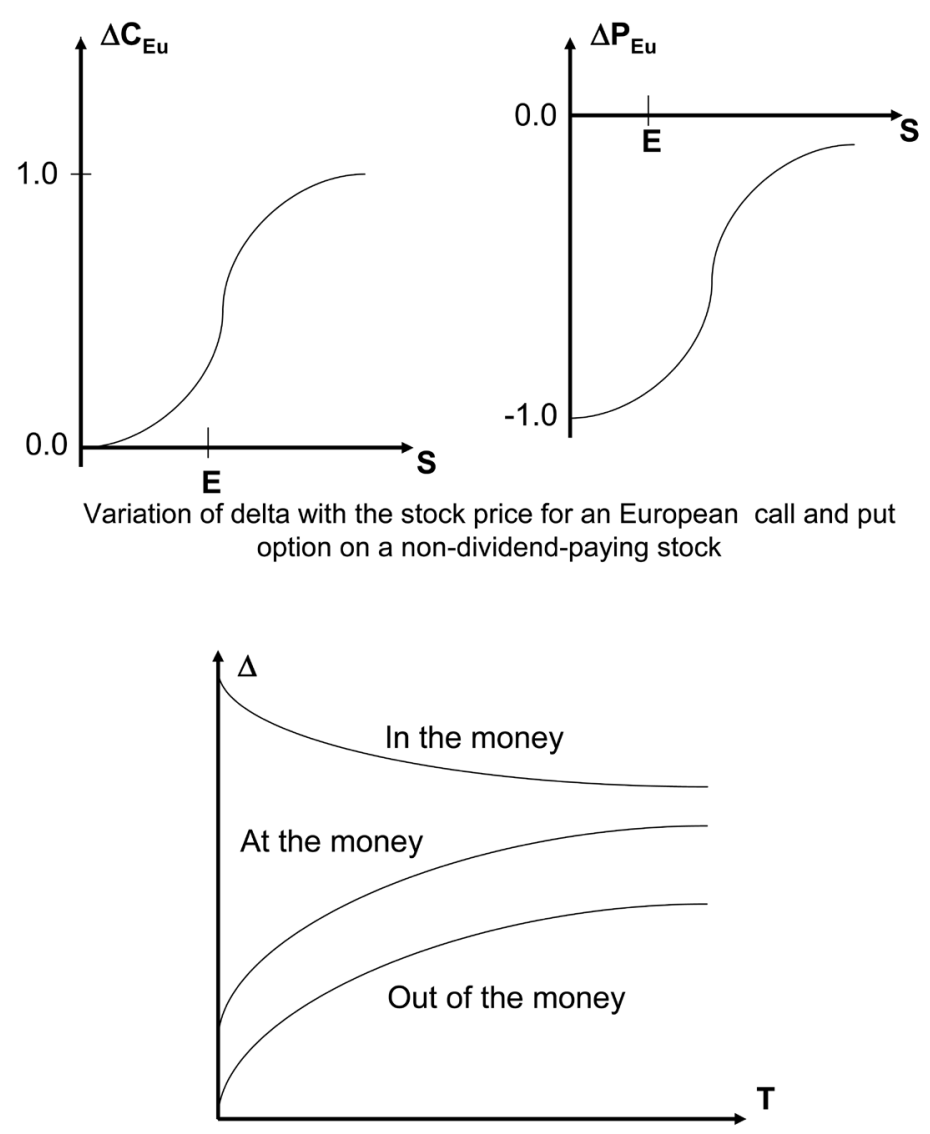

Variation of delta with the time to maturity for an European call option

Figure 3. Variation of $\Delta$ with respect to European options. 
for a put option, and

$$
S_{1} \leq S_{2} \Rightarrow C_{E u}\left(S_{1}, t\right) \leq C_{E u}\left(S_{2}, t\right) \Rightarrow \frac{\partial C_{E u}}{\partial S} \geq 0
$$

for a call option.

Therefore, in the case of a put option, which is important for this analysis, we have $\partial P_{E u} / \partial S \leq 0$.

\section{Lemma 1}

$$
\int_{0}^{T_{S}-t} \frac{1}{I_{\rho}} \frac{\partial \rho}{\partial S} \frac{\partial V_{E u}}{\partial S} \mathrm{~d} \tau \leq 0
$$

\section{Proof.}

We proceed by contradiction.

For any $S_{0}$, it holds that

$$
\int_{0}^{T_{S}-t} \frac{1}{I_{\rho}} \frac{\partial \rho}{\partial S} \frac{\partial V_{E u}}{\partial S} \mathrm{~d} \tau>0
$$

By continuity, the integral remains being positive for any arbitrary and small $\delta$ such that $S \in\left[S_{0}-\delta\right.$, $\left.S_{0}+\delta\right]$, then

$$
\begin{aligned}
& \int_{S_{0}-\delta}^{S_{0}+\delta} \int_{0}^{T_{S}-t} \frac{1}{I_{\rho}} \frac{\partial \rho}{\partial S} \frac{\partial V_{E u}}{\partial S} \mathrm{~d} \tau \mathrm{d} S=\int_{0}^{T_{S}-t} \int_{S_{0}-\delta}^{S_{0}+\delta} \frac{1}{I_{\rho}} \frac{\partial \rho}{\partial S} \frac{\partial V_{E u}}{\partial S} \mathrm{~d} S \mathrm{~d} \tau \\
& =\int_{0}^{T_{S}-t}\left[-\int_{S_{0}-\delta}^{S_{0}+\delta} \rho \frac{\partial^{2} V_{E u}}{\partial S^{2}} \mathrm{~d} S\right] \mathrm{d} \tau+\int_{0}^{T_{S}-t}\left[\left(\rho \frac{\partial V_{E u}}{\partial S}\right)_{S_{0}-\delta}^{S_{0}+\delta}\right] \mathrm{d} \tau \\
& +\int_{0}^{T_{S}-t}\left[\int_{S_{0}-\delta}^{S_{0}+\delta} \frac{1}{\left(I_{\rho}\right)^{2}} \frac{\partial V_{E u}}{\partial S}\left(\int_{t+\tau}^{T_{S}} \frac{\partial \rho}{\partial S} \mathrm{~d} k\right) \mathrm{d} S\right] \mathrm{d} \tau \\
& =\int_{0}^{T_{S}-t}\left(I_{1}+I_{2}+I_{3}\right) \mathrm{d} \tau .
\end{aligned}
$$

Now, we analyze each term separately.

$$
I_{2}=\left(\rho \frac{\partial V_{E u}}{\partial S}\right)_{S_{0}-\delta}^{S_{0}+\delta}=\rho\left(S_{0}+\delta, t\right) \frac{\partial V_{E u}\left(S_{0}+\delta, t\right)}{\partial S}-\rho\left(S_{0}-\delta, t\right) \frac{\partial V_{E u}\left(S_{0}-\delta\right)}{\partial S} \rightarrow 0
$$

The difference $I_{2}$ tends to zero, because $\delta$ is arbitrary and the functions involved are continuous, so that it can be taken as small as one likes, which makes the difference negligible.

For the term $I_{1}$, we have that $\rho \geq 0$ since it is a density function and on the other side we had that $\partial V_{E u} / \partial S=N\left(d_{1}\right)-1 \leq 0$, then

$$
\frac{\partial^{2} V_{E u}}{\partial S^{2}}=N^{\prime}\left(d_{1}\right) \geq 0 \text { con } N^{\prime}\left(d_{1}\right)=\frac{1}{\sqrt{2 \pi}} \mathrm{e}^{-\left(d_{1}\right)^{2} / 2} .
$$

So $\rho\left(\partial V_{E u} / \partial S\right) \geq 0$ and

$$
I_{1}=-\int_{S_{0}-\delta}^{S_{0}+\delta} \rho \frac{\partial^{2} V_{E u}}{\partial S^{2}} \mathrm{~d} S \leq 0 .
$$

For $I_{3}$ in (29) we know that $\partial V_{E u} / \partial S$ is negative for a put option and $\partial \rho / \partial S<0$, so

$$
I_{3}=\int_{S_{0}-\delta}^{S_{0}+\delta} \frac{1}{\left(I_{\rho}\right)^{2}} \frac{\partial V_{E u}}{\partial S}\left(\int_{t+\tau}^{T_{S}} \frac{\partial \rho}{\partial S} \mathrm{~d} k\right) \mathrm{d} S \geq 0
$$

Although the term is greater than zero, we can make this smaller that the negative terms. This is explained with detail in remark 5.

Finally, we obtain 


$$
\int_{0}^{T_{S}-t}\left(I_{1}+I_{2}+I_{3}\right) \mathrm{d} \tau \leq 0 .
$$

Which is in contradiction with (28).

We therefore have the Lemma 1

$$
\int_{0}^{T_{S}-t} \frac{1}{I_{\rho}} \frac{\partial \rho}{\partial S} \frac{\partial V_{E u}}{\partial S} \leq 0 .
$$

Moreover, $\rho\left(S, T_{S}-t\right) \geq 0$ and $V_{E u}\left(S, T_{S}\right) \geq 0$ so that

$$
-\rho\left(S, T_{S}-t\right) V_{E u}\left(S, T_{S}\right) \leq 0 .
$$

Remark 5 It is important to note that the time $T_{S}$ depends on the free boundary $S_{f}$, which implies that the first and second derivatives with respect to $T_{S}$ also depend on the free boundary. But we know that the free boundary is regular, concave, increasing and at least of class $C^{2}$, which guarantees that this boundary and in turn the derivatives of $T_{S}$ also exist.

Remark 6 For the positive terms of inequality (25), let $m$ be an arbitrary large value such that

$$
\int_{t+\tau}^{T_{S}} \rho(S, k) \mathrm{d} k=m .
$$

This is possible because the Fokker-Planck equation is linear and we can always rescale the solution. Also note that the valuation formula is linear with respect to $\rho$. Therefore we can multiply and divide by $m$ without changing the result. In the calculation presented above, as well as in inequality (25), the positive terms scale at least as $1 / I_{\rho}$ and therefore can be made smaller in relation to the negative terms that are invariant under such rescaling. Notice that, there is at least one of those terms, for example, the second one in inequality (25), $-\rho\left(S, T_{S}-t\right) V_{E u}\left(S, T_{S}\right)$.

With that we have finally proved the claim that the new way to price American options given by (19) coincides with the standard one.

\section{Analytical Comparison with Other Methodologies}

Another way to see the consistency of our proposal is to verify that the formula of Carr, Jarrow and Myneni [5] and the binomial tree method are consistent with our formula 19.

1) Carr, Jarrow and Myneni Formula

Using their notation, in a region $C \equiv\left(b_{t}, \infty\right) \times[0, T]$, the American put option value, $P_{\tau}$, can be decomposed into the price of a European put option, $p_{\tau}$, and an early exercise premium, $e_{\tau}$ :

$$
\begin{gathered}
P_{t}=p_{t}+e_{t} \\
e_{t}=r E \int_{t}^{T} \mathrm{e}^{-r \tau} N\left(\frac{\ln \left(B_{\tau} / S\right)-\rho_{2} \tau}{\sigma \sqrt{\tau}}\right) \mathrm{d} \tau,
\end{gathered}
$$

and $N(x) \equiv \int_{0}^{x} \exp \left(-z^{2} / 2\right) / \sqrt{2 \pi} \mathrm{d} z$ is the standard normal distribution function. $B_{t}$ is the free boundary and $\rho_{2}=r-\sigma / 2$.

Then starting from the previous theorem we have:

$$
\begin{aligned}
P_{t} & =p_{t}+e_{t}=p_{t}+r E \int_{t}^{T} \mathrm{e}^{-r \tau} N\left(\frac{\ln \left(B_{\tau} / S\right)-\rho_{2} \tau}{\sigma \sqrt{\tau}}\right) \mathrm{d} \tau \\
& =\int_{t}^{T} 2 \delta(\tau-t) p_{\tau} \mathrm{d} \tau+\int_{t}^{T} r E p_{\tau} \frac{Z_{\tau}}{p_{\tau}} \mathrm{d} \tau \\
& =\int_{t}^{T} p_{\tau}\left[2 \delta(\tau-t)+r E \frac{Z_{\tau}}{p_{\tau}}\right] \mathrm{d} \tau=\int_{t}^{T} p_{\tau} \rho_{n}(S, \tau) \mathrm{d} \tau .
\end{aligned}
$$

Here, $p_{t}=P_{E u}(S, t), \quad z_{\tau}=\mathrm{e}^{-r \tau} N\left(\frac{\ln \left(B_{\tau} / S\right)-\rho_{2} \tau}{\sigma \sqrt{\tau}}\right)$ and $\rho_{n}(S, \tau)=2 \delta(\tau-t)+r E \frac{z_{\tau}}{p_{\tau}}$. 
2) Binomial Tree Method

It is easy to check that the standard binomial tree method can be decomposed in two steps. First, we find the exercise (free) boundary and substitute the prices by the payoff. Second, we proceed exactly as in the European case restricted to this region. This is precisely the discrete analogue of expression (19).

\subsection{Application of the Alternative Formula}

For verifying the efficiency of the new formula to price American options it is necessary to analyze its behavior with specific examples. For that we consider several cases, in which we estimate the American option prices and we compare those with the prices obtained with other methods, such as: the tree method (Cox, Ingersoll and Rubinstein), the finite difference method (implicit and explicit) and Longstaff and Schwartz method. For consistency, we make all calculations for the log-normal case (Black-Scholes).

Before going into details about the numerical methods, we present the algorithm by which we calculate the prices of American put options.

For an $S \in\left[0, S_{\max }\right]$ with $S_{\max }=2 S$ fixed:

1) In general, the probabilities are estimated using the solution of the partial differential equation (15) for various times before or equal to expiring. These probabilities for the log-normal case can be found also through the explicit solution (17).

2) Probabilities are normalized so that $\int_{0}^{t} \rho_{n}(S, \tau) \mathrm{d} \tau=1$.

3) Calculate the price of a European put option by the explicit Black-Scholes formula with underlaying asset $S$ in different real times $t^{8}$.

4) a) If a priori we know the free boundary, $P=P_{E u}$ in the non early exercise region.

b) If the free boundary is not known, we check whether the early exercise is optimal at each time step, i.e., takes the maximum between the solution of the Black-Scholes equation $\left(P_{E u}(S, t)\right)$ and the payoff function, $\max (S, 0)$. In other words we consider the function

$$
P(t)=\max \left(P_{E u}(S, t), \max (E-S, 0)\right) .
$$

5) Multiply the normalized probability, $\rho$, by the function $P(t)$ for every $t$.

6) Calculate the integral $\int_{t}^{T_{S}} \rho_{n}(S, \tau) P(S, \tau) \mathrm{d} \tau$ to determine the value of an American put option.

The flow chart for the algorithm is given in Figure 4.

With this algorithm, we describe the numerical way to solve specific examples and we compare the results, evaluated with traditional methods and our approach.

To find the numerical solution of the Fokker-Planck PDE, use the Finite Element Modeling Laboratory package (FEMLAB Multiphysics in MATLAB), which is an advanced software package for modeling and simulating many physical process that can be described through PDE's.

For the particular case, where $S$ follows a log-normal process, we can use the explicit solution (15) to determine the probabilities $\rho$. The Table 1 shows the explicit solution for these probabilities and the corresponding numerical approximations. We consider $S_{0}=80, r=0.1, \sigma=0.4$ and $\Delta t=0.0833$ for the twelve months of a year.

The numerical approach is very close to the explicit probabilities. So we use both interchangeably. For practical purposes and only in this case, to verify that the new way to value American options (19) is consistent with the log-normal case (Black-Scholes), we use the closed formula (17), which was implemented in MATLAB.

Now, we show a concrete example according to the steps of the algorithm for pricing an American option with formula (19).

Let the asset price $S_{0}=50$, the exercise price $E=50$, the riskless interest rate $r=0.1$, the volatility $\sigma=0.4$ and the time $t=5$ periods, which correspond to $t=0.41667 \quad(t=T-\tau)$. In this case, we do not know the location of the free boundary. The results are showed in the Table 2.

\footnotetext{
${ }^{8} \mathrm{~A}$ priori we do not know the location of the free boundary, which implies that the value of expiration time at the boundary $T_{S}$ is unknown, then for numerical calculations instead we take the expiry time $T$.
} 


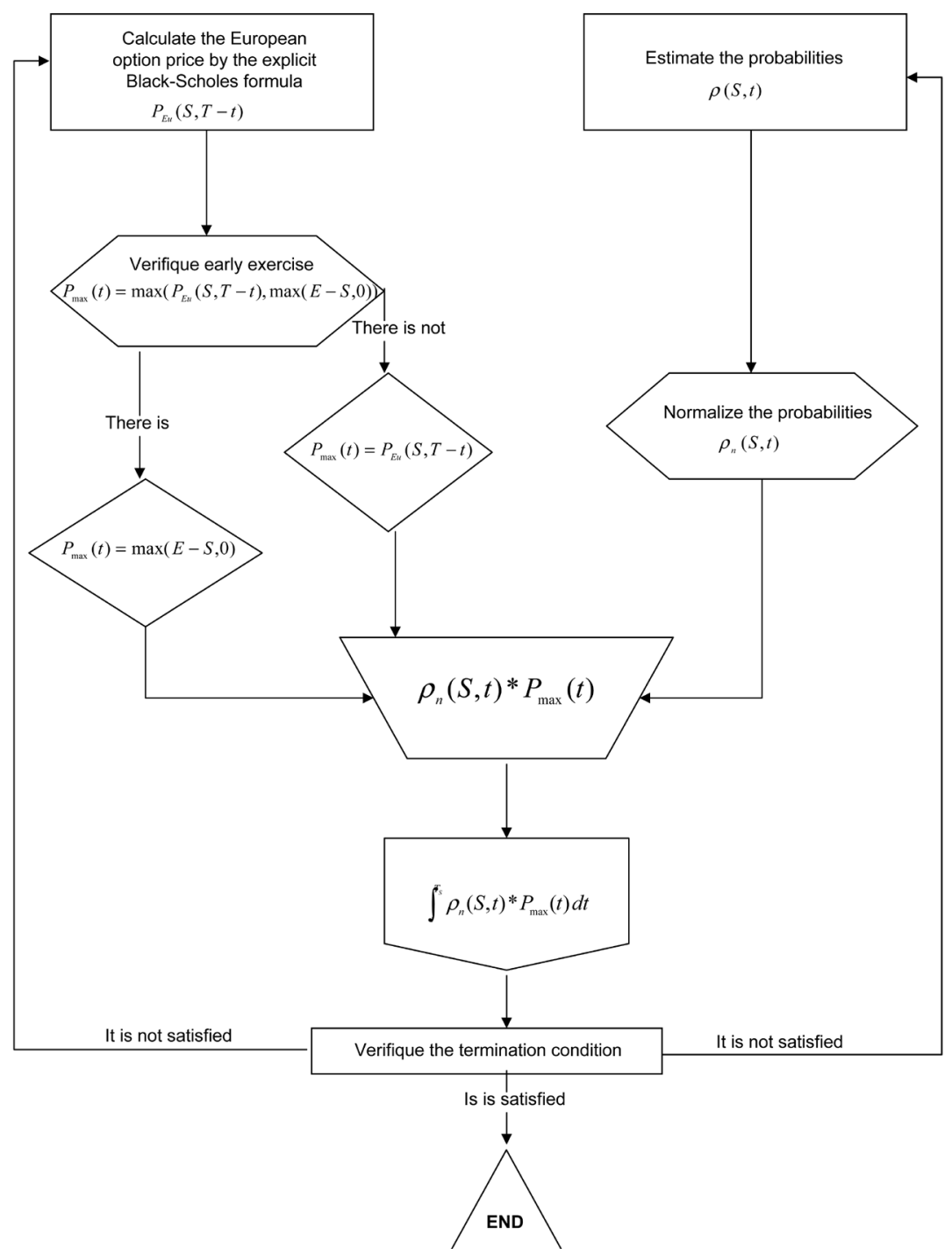

Figure 4. Flow chart to find the price of an American put option given by the formula (19).

Table 1. Explicit and numerical comparison of the probabilities $(\rho)$.

\begin{tabular}{ccc}
\hline$t_{\text {months }}$ & explicit $\rho$ & numerical $\rho$ (EDP) \\
1 & 0.0432 & 0.0429 \\
2 & 0.0305 & 0.0302 \\
3 & 0.0249 & 0.0245 \\
4 & 0.0216 & 0.0210 \\
5 & 0.0193 & 0.0189 \\
6 & 0.0176 & 0.0173 \\
7 & 0.0163 & 0.0161 \\
8 & 0.0153 & 0.0151 \\
9 & 0.0144 & 0.0143 \\
10 & 0.0136 & 0.0135 \\
11 & 0.0130 & 0.0129 \\
12 & 0.0125 & 0.0124 \\
\hline
\end{tabular}


Table 2. American put option values.

\begin{tabular}{cl}
\hline$t_{\text {months }}$ & $V_{A}(S, t)$ \\
\hline 1 & 5.3929 \\
2 & 5.3815 \\
3 & 5.3675 \\
4 & 5.3508 \\
5 & 5.3315 \\
\hline
\end{tabular}

Source: Own calculus using, as approach to the integral, the trapezoid method.

\subsection{Comparison with Other Numerical Methods}

In the following examples, we estimate the American put option values by the binomial method and the finite differences (implicit and explicit), we take the Bern Arne numerical implementation Ødegaard (see [33]), and for other methods CRR (an American option price by Cox-Ross-Rubinstein tree method calculated using the finite differences for the Black-Scholes PDE)and FD (American option price by finite differences applying to the Black-Scholes PDE). The numerical implementations of these methods were made in MATLAB (see [34]). The New a) for boundary know, and b) for boundary not know method corresponds to our methodology for evaluating an American put option with (19). For more details on these methods, see [14] [35].

In Table 3, in each example all involved variables are changed in the model and we do not know $T_{S}$, we take it as $T .^{9}$

If in Example 3) we consider $t=0.1$ and $T=0.5833$, then 1) is equal to 5 and New 2) equals 4.7936. For the case that $t=0.4$ and $T=0.5833$, posted a) is 5 and New b) is 4.8434 .

Finally, in Table 4 we show the formula New a) and New b) when the time changes. We can see that when the boundary is known, the price does not change. However, whether the boundary is not known the price changes and it is bigger whether $t$ goes up.

There are other more complex numerical methods to estimate american options, within them we mention some: [3] [4] [8] [11] [27] [36]-[41].

\section{Conclusions}

In conclusion we can say the following:

Mathematically, we prove that this new proposal for pricing American options is consistent with the BlackScholes approach (canonical problem).

One application of this formula could be seen in the analytical proof that an American call option is equal to a European call option, i.e., the early exercise of an American call option is not optimal and the probability $\rho$ is a Dirac delta concentrated on $\{t=T\}$. This result is shown in [31].

As an application of the new way of pricing American options, we show numerically several examples. This proposal gives adequate proxies in comparison with traditional methods, such as binomial trees (Binomial CRR) and finite differences (Explicit and Implicit).

The advantage of using this new formula is that in order to compute the price of any American option, we only need to approximate the value of the probability of staying in the requested state, provided we already know the location of the free boundary, as the value of a European put option is known explicitly, which makes it more flexible and easy to estimate.

\footnotetext{
${ }^{9}$ The time is annual, namely, 5 months is equivalent to $5 / 12=0.4167 . t_{m}=1,2, \cdots, 12$ is the monthly time where the option is estimated in the New method. In the other methods (binomial, explicit, implicit, CRR, FD and LSM), the time corresponding is where $T=1$ ( $\approx 12$ months). $S=S_{0}$ and $S_{\max }=2 S_{0}$. Consider $N=100$ for the time and $M=100$ for the asset, except in the explicit method. In this method, we use $N=11$ and $M=20$ in the examples. $\Delta t=(t / 12) / N$ and $\Delta S=(2 S) / M$.
} 
Table 3. Comparison of an American put option.

\begin{tabular}{cccc|}
\hline Variables & Example 1 & Example 2 & Example 3 \\
\hline$S_{0}$ & 300 & 62 & 40 \\
$E$ & 300 & 60 & 45 \\
$r$ & 0.08 & 0.10 & 0.05 \\
$\sigma$ & 0.3 & 0.2 & 0.2 \\
$t_{m}$ & 4 & 5 & 7 \\
\hline Method & & $V_{A}(S, t)$ & 5.0000 \\
\hline New a) & 23.1808 & 1.6929 & 4.8539 \\
New b) & 23.1808 & 1.6929 & 5.1420 \\
Binomial & 22.9975 & 1.7652 & 5.0550 \\
Explicit & 22.8051 & 1.6504 & 5.1340 \\
Implicit & 22.9190 & 1.7535 & 5.4720 \\
CRR & 22.9970 & 1.7652 & 5.4030 \\
\hline FD & 22.4680 & 1.5100 & \\
\hline
\end{tabular}

Table 4. Comparison of an American put option.

\begin{tabular}{ccc}
\hline Initial time $t$ & New a) & New b) \\
\hline$t=0$ & 3.000 & 2.812 \\
$t=0.1$ & 3.000 & 2.835 \\
$t=0.4$ & 3.000 & 2.9351 \\
\hline
\end{tabular}

Moreover, by obtaining the transition probabilities via the Kolmogorov equation, we have the advantage of being able to systematically incorporate the effect of macroeconomic factors, which would make the calculation of the option price more realistic.

\section{Acknowledgements}

The authors thank the Editor and the referee for their comments. The authors also appreciate the valuable comments and suggestions of Josué Cortés, Gonzalo Rangel, Patricia Saavedra, Alvaro Cartea, Gilberto Flores and the seminar participants in Banco de México. The views and conclusions presented in this paper are exclusively the responsibility of the authors and do not necessarily reflect those of Banco de México.

\section{References}

[1] Bank, P. and Föllmer, H. (2002) American Options, Multi-Armed Bandits, and Optimal Consumption Plans: A Unifying View. Paris-Princeton Lectures on Mathematical Finance, 1814, 1-42. http://dx.doi.org/10.1007/978-3-540-44859-4_1

[2] Bladt, M. and Rydberg, T.H. (1998) An Actuarial Approach to Option Pricing under the Physical Measure and without Market Assumptions. Insurance: Mathematics and Economics, 22, 65-73. http://dx.doi.org/10.1016/s0167-6687(98)00013-4

[3] Bally, P., Pages, G. and Printems, J. (2005) A Quantization Tree Method for Pricing and Hedging Multidimensional American Options. Mathematical Finance, 15, 119-168. http://dx.doi.org/10.1111/j.0960-1627.2005.00213.x

[4] Broadie, M. and Cao, M.H. (2008) Improved Lower and Upper Bound Algorithms for Pricing American Options by Simulations. Quantitative Finance, 8, 845-861. http://dx.doi.org/10.1080/14697680701763086

[5] Carr, P., Jarrow, R. and Myneni, R. (2006) Alternative Characterizations of American Put Options. Mathematical Finance, 2, 87-106. http://dx.doi.org/10.1111/j.1467-9965.1992.tb00040.x

[6] Geske, R. and Johnson, H.E. (1984) The American Put Option Valued Analytically. The Journal of Finance, 39, 15111524. http://dx.doi.org/10.1111/j.1540-6261.1984.tb04921.x

[7] Han, H.D. and Wu, X.N. (2004) A Fast Numerical Method for the Black-Scholes Equation of American Options. SIAM Journal on Numerical Analysis, 41, 2081-2095. 
[8] Ikonen, S. and Toivanen, J. (2008) Efficient Numerical Methods for Pricing American Options Under Stochastic Volatility. Numerical Methods for Partial Differential Equations, 24, 104-126. http://dx.doi.org/10.1002/num.20239

[9] Merton, R.C., Foreword by Samuelson, P.A. (1995) Continuous-Time Finance. Blackwell, Massachusetts.

[10] Musiela, M. and Rutkowski, M. (1998) Martingale Methods in Financial Modelling. Springer-Verlag, Berlin.

[11] Tangman, D.Y., Gopaul, A. and Bhuruth, M. (2008) A Fast High-Order Finite Difference Algotithm for Pricing American Options. Journal of Computational and Applied Mathematics, 222, 17-29. http://dx.doi.org/10.1016/j.cam.2007.10.044

[12] Jia, Q. (2009) Pricing American Options using Monte Carlo Methods. Department of Mathematics Uppsala University, U.U.D.M. Project Report. http://www2.math.uu.se/research/pub/Jia1.pdf

[13] Uys, N. (2005) Optimal Stopping Problems and American Option. Master of Science Dissertation Submitted to the Faculty of Science, University of the Witwatersrand, Johannesburg.

[14] Wilmott, P., Dewynne, J. and Howison, S. (1993) Option Pricing: Mathematical Models and Computation. Oxford Financial Press, Oxford.

[15] Levendorskii, S. (2004) The American Put and European Options Near Expiry, Under Lévy Processes. Department of Mathematics, University of Leicester, Leicester, 1-30. http://dx.doi.org/10.2139/ssrn.520062

[16] Hyungsok, A. and Wilmott, P. On Trading American Options. OCIAM, Oxford University, Oxford. http://citeseerx.ist.psu.edu/viewdoc/download?doi=10.1.1.38.7270\&rep=rep1\&type=pdf

[17] Luenberger, D.G. (1998) Investment Science. Oxford University Press, Oxford.

[18] Méndez, R.E. (2007) Correlación Temporal en la Valuación de Derivados. Tesis de Maestra en Ciencias Matemáticas, UNAM, Mexico City.

[19] Risken, H. (1989) The Fokker-Planck Equation: Methods of Solution and Applications. Springer-Verlag, Berlin. http://dx.doi.org/10.1007/978-3-642-61544-3

[20] Werner, H. and Lefever, R. (1984) Noise-Induced Transitions: Theory and Applications in Physics, Chemistry and Biology. Springer-Verlag, Berlin.

[21] Carr, P. and Hirsa, A. (2002) Why Be Backward? Forward Equations for American Options. Morgan Stanley/Courant Institute, NYU, New York, 1-25.

[22] Evans, L. (1998) Partial Differential Equations. American Mathematical Society, Providence.

[23] Lyuu, L. (2000) Financial Engineering and Computation: Principles, Mathematics and Algorithms. Cambridge University Press, New York.

[24] Pontryagin, L., Andronov, A. and Vitt, A. (1989) Appendix: On the Statistical Treatment of Dynamical Systems. Springer-Verlag, New York, 329-348.

[25] Zhang, W.-B. (1991) Synergetic Economics: Time and Change in Nonlinear Economics. Springer-Verlag, Berlin.

[26] Llenera-Garcés, F. (2000) Una Nota Sobre Valoración de Opciones Americanas y Arbitraje. Investigaciones Económicas, XXIV, 207-218.

[27] Mikosch, T. (1999) Elementary Stochastic Calculus with Finance in View. World Scientific Publishing, Singapore.

[28] Steele, J.M. (2001) Stochastic Calculus and Financial Applications. Springer-Verlag, New York. http://dx.doi.org/10.1007/978-1-4684-9305-4

[29] Padilla, P. and Bladt, M. (2001) Nonlinear Financial Models: Finite Markov Modulation and Its Limits. In: Avellaneda, M., Ed., Quantitative Analysis in Financial Markets, Collected Papers of the New York University Mathematical Finance Seminar, Vol. III, World Scientific Publishing, Singapore, 159-171.

[30] Elizondo, R. (2009) Incorporación de Factores Macroeconómicos en los Modelos de Valuación de Productos Derivados. Thesis de Doctorado en Ciencias, IIMAS, UNAM, Mexico City.

[31] Elizondo, R. and Padilla, P. (2008) An Analytical Approach to Merton's Rational Option Pricing Theory. Analysis and Application, 6, 169-182. http://dx.doi.org/10.1142/s0219530508001110

[32] Jarrow, R.A. (1998) Preferences, Continuity and the Arbitrage Pricing Theory. The Review of Financial Studies, 1, 159-172. http://dx.doi.org/10.1093/rfs/1.2.159

[33] Odegaard, B.A. (2007) Financial Numerical Recipes in C++. http://www1.uis.no/ansatt/odegaard/gcc_prog/recipes/recipes.pdf

[34] Numerical Implementation Website. http://www.mathworks.com/matlabcentral/fileexchange/loadFile.do?objectIdB

[35] Hull, J. (2000) Options, Futures, and Other Derivatives. Prentice Hall, Upper Saddle River.

[36] Broadie, M. and Detemple, J. (1996) American Option Valuation: New Bounds, Approximations and a Comparison of 
Existing Methods. The Review of Financial Studies, 9, 1211-1250. http://dx.doi.org/10.1093/rfs/9.4.1211

[37] Chesney, M. and Jeanblanc, M. (2003) Pricing American Currency Options in a Jump Diffusion Model. 1-19.

[38] Christ Churh College (2004) Nonlinear Black Scholes Modelling: FDM vs FEM. A Thesis Submitted in Partial Fulfilment of the Requirements for the MSc in Mathematical Finance, Oxford University, Oxford.

[39] Leung, L.T. and Po-Shing, W.S. (2002) Valuation of American Options via Basis Functions. Department of Statistics, Stanford University, Technical Report No. 2002-28, 1-29.

[40] Longstaff, F. and Schwartz, E. (2001) Valuing American Options by Simulation: A Simple Least-Squares Approach. This Paper Is Posted at the Scholarship Repository, University of California, Oakland. http://repositories.edlib.org/anderson/fin/1-01

[41] Matache, A.M., Nitsche, P.A. and Schwab, C. (2003) Wavelet Galerkin Pricing of American Options on Lévy Driven Assets. Research Report No. 2003-06, Zürich, 1-26. 